\section{Fast Positive Deconvolution of Hyperspectral Images}

\author{
Simon Henrot, Charles Soussen, David Brie
}

\begin{abstract}
In this correspondence, we provide an efficient scheme for performing deconvolution of large hyperspectral images under a positivity constraint, while accounting for spatial and spectral smoothness of the data.
\end{abstract}

Index Terms-Image restoration, hyperspectral images, regularized least squares, positivity, cross-spectral prior information

\section{INTRODUCTION}

In many imaging applications, the acquisition process induces various degradations on the collected image which can prevent accurate post-processing of the data of interest. For instance, the image of a biological sample by a microscope is blurred due to diffraction and corrupted by stochastic noise mainly caused by photon-to-electron conversion [1]. Restoration methods such as deconvolution are thus used whenever post processing requires it.

Dealing with hyperspectral images (that is, a stack of images representing the same scene captured at many wavelengths) adds the challenge of accounting for cross-spectral information within the restoration process [2], [3]: the intensity values of a given pixel at two neighboring wavelengths should be close. When the data size is large, advanced deconvolution methods become computationally prohibitive and linear methods such as Tikhonov or Wiener-filtering can be used to process the set of images [4], [5]. Within this framework, the problem is formulated as the minimization of a compound criterion which possesses a closed-form solution. Up to the circular approximation of the observation matrix, this solution can be efficiently computed in the Fourier domain using 2D Fast Fourier Transforms (FFTs) [6].

A drawback of these methods is their inability to restore the original object's high frequency components beyond the PSF bandwidth. This typically results in ringing artifacts around edges which can generate negative-valued pixels in the deconvolved image [7]. When the image is known to be positive (e.g., acquired by a photon counting process), a positivity constraint needs to be applied to yield a physically meaningful estimate. In [8], the authors use the augmented Lagrangian method to solve this constrained optimization problem for $2 \mathrm{D}$ images ; another fast $2 \mathrm{D}$ restoration method using variable splitting is given in [9]. Other popular algorithms are the Image Space Reconstruction Algorithm (ISRA) in the case of Gaussian noise and the Richardson-Lucy algorithm for Poisson noise: these methods can be applied to the hyperspectral positive restoration problem but do not lead to fast computations [10].

To the best of our knowledge, no hyperspectral restoration algorithm accounts for both cross-spectral information and positivity in a fast manner. The main contribution of this paper is to propose an algorithm that incorporates these two priors while remaining computationally efficient.

The remainder of this paper is organized as follows. Section II introduces the observation model and notations used throughout the paper. The deconvolution problem is formulated in section III. In section IV, we detail the procedure we use to solve the problem. Section V presents experimental results on synthetic data and some insight into the influence of the hyperparameters. Finally, we conclude in section VI.

This work was supported by the French Agence Nationale de la Recherche, with reference number ANR-09-BLAN-0336-04. The authors are with the Université de Lorraine and the CNRS at the CRAN (Research Center for Automatic Control), UMR 7039, Campus Sciences, BP 70239, 54506 Vandœuvre cedex, France, e-mail: firstname.lastname@cran.uhp-nancy.fr.

\section{OBSERVATION MODEL AND NOTATIONS}

The hyperspectral image to be restored $\mathbf{X}$ is assumed to be a stack of $N$ matrices $\left\{\mathbf{X}_{\ell}, \ell=1, \ldots, N\right\}$ of size $M \times M$ (we use square matrices for simplicity and without loss of generality), where subscript $\ell$ refers to the wavelengths or channels. Using lexicographical order, the stack can be reshaped into a collection of $M^{2} \times 1$ vectors $\left\{\mathbf{x}_{\ell}, \ell=1, \ldots, N\right\}$ or a single $M^{2} N \times 1$ vector $\mathbf{x}$ with $\mathbf{x}^{t}=\left[\mathbf{x}_{1}^{t}, \ldots, \mathbf{x}_{N}^{t}\right]$ and we use similar notations for other images.

\section{A. Within-channel observation model}

Within a given channel $\ell, \mathbf{X}_{\ell}$ generates data $\mathbf{Y}_{\ell}$ according to the two-dimensional (2D) observation model (see for instance [11])

$$
\mathbf{Y}_{\ell}=\mathcal{H}_{\ell} \underset{2 D}{*} \mathbf{X}_{\ell}+\mathbf{N}_{\ell}
$$

where $\mathcal{H}_{\ell}$ is the $M \times M$ convolution kernel (possibly comprising null entries) encoding the $\ell$-th channel Point-Spread Function (PSF):

$$
\mathcal{H}_{\ell}=\left(\begin{array}{ccc}
\mathcal{H}_{11}^{\ell} & \ldots & \mathcal{H}_{1 M}^{\ell} \\
\vdots & \ddots & \vdots \\
\mathcal{H}_{M 1}^{\ell} & \ldots & \mathcal{H}_{M M}^{\ell}
\end{array}\right),
$$

$*_{2 D}$ denotes the discrete $2 \mathrm{D}$ convolution product carried out in the image domain, yielding an output of size $M \times M$ and $\mathbf{N}_{\ell}$ is a noise term assumed to be independent and identically distributed (i.i.d) and Gaussian. Equation (1) rewrites as a linear system:

$$
\mathbf{y}_{\ell}=\mathbf{H}_{\ell} \mathbf{x}_{\ell}+\mathbf{n}_{\ell}
$$

where $\mathbf{H}_{\ell}$ is a $M^{2} \times M^{2}$ block-Toeplitz matrix with $M \times M$ Toeplitz blocks, e.g. for $M=3, \mathbf{H}_{\ell}$ reads

$$
\mathbf{H}_{\ell}=\left[\begin{array}{ccc}
\mathbf{H}_{\mathbf{2}}^{\ell} & \mathbf{H}_{\mathbf{1}}^{\ell} & \mathbf{0} \\
\mathbf{H}_{\mathbf{3}}^{\ell} & \mathbf{H}_{\mathbf{2}}^{\ell} & \mathbf{H}_{\mathbf{1}}^{\ell} \\
\mathbf{0} & \mathbf{H}_{\mathbf{3}}^{\ell} & \mathbf{H}_{\mathbf{2}}^{\ell}
\end{array}\right] \text { with } \mathbf{H}_{\mathbf{i}}^{\ell}=\left(\begin{array}{ccc}
\mathcal{H}_{2 i}^{\ell} & \mathcal{H}_{1 i}^{\ell} & 0 \\
\mathcal{H}_{3 i}^{\ell} & \mathcal{H}_{2 i}^{\ell} & \mathcal{H}_{1 i}^{\ell} \\
0 & \mathcal{H}_{3 i}^{\ell} & \mathcal{H}_{2 i}^{\ell}
\end{array}\right)
$$

Working assumption: We impose periodic boundary conditions on PSFs $\mathcal{H}_{\ell}$ 's. This approximation allows $\mathbf{H}_{\ell}$ to be rewritten as a blockcirculant matrix with circulant blocks [5], a structure we will denote by circulant-block-circulant (CBC):

$$
\mathbf{H}_{\ell}=\left[\begin{array}{lll}
\mathbf{H}_{\mathbf{2}}^{\ell} & \mathbf{H}_{\mathbf{1}}^{\ell} & \mathbf{H}_{\mathbf{3}}^{\ell} \\
\mathbf{H}_{\mathbf{3}}^{\ell} & \mathbf{H}_{\mathbf{2}}^{\ell} & \mathbf{H}_{\mathbf{1}}^{\ell} \\
\mathbf{H}_{\mathbf{1}}^{\ell} & \mathbf{H}_{\mathbf{3}}^{\ell} & \mathbf{H}_{\mathbf{2}}^{\ell}
\end{array}\right] \text { with } \mathbf{H}_{\mathbf{i}}^{\ell}=\left(\begin{array}{ccc}
\mathcal{H}_{2 i}^{\ell} & \mathcal{H}_{1 i}^{\ell} & \mathcal{H}_{3 i}^{\ell} \\
\mathcal{H}_{3 i}^{\ell} & \mathcal{H}_{2 i}^{\ell} & \mathcal{H}_{1 i}^{\ell} \\
\mathcal{H}_{1 i}^{\ell} & \mathcal{H}_{3 i}^{\ell} & \mathcal{H}_{2 i}^{\ell}
\end{array}\right)
$$

A well-known property of $\mathrm{CBC}$ matrices is that they can be diagonalized by a two-dimensional discrete Fourier transform (2D-DFT): this property is used in section IV.

\section{B. Hyperspectral observation model}

The hyperspectral observation model reads $\mathbf{y}=\mathbf{H x}+\mathbf{n}$ where the $N M^{2} \times N M^{2}$ observation matrix $\mathbf{H}$ is block-diagonal since imaging in a given channel $\ell$ does not interfere with other channels $\ell^{\prime} \neq \ell$ :

$$
\mathbf{H}=\left[\begin{array}{cccc}
\mathbf{H}_{1} & \mathbf{0} & \ldots & \mathbf{0} \\
\mathbf{0} & \ddots & \ddots & \vdots \\
\vdots & \ddots & \ddots & \mathbf{0} \\
\mathbf{0} & \ldots & \mathbf{0} & \mathbf{H}_{N}
\end{array}\right] .
$$

We further assume that the noise process $\mathbf{n}_{\ell}$ for a given channel is independent of the other channels $\ell^{\prime} \neq \ell$, and that the noise variance is common to all channels. Using this assumption, the noise process $\mathbf{n}$ (accounting for all channels together) obeys an i.i.d. Gaussian distribution. 


\section{PROBLEM FORMULATION}

\section{A. Linear restoration problem}

The deconvolution problem can be formulated as the estimation of $\mathbf{x}$ given data $\mathbf{y}$ and the system response $\mathbf{H}$. In the framework of least square restoration [5], it is usually written as the unconstrained minimization of a composite criterion

$$
\mathcal{J}(\mathbf{x})=\|\mathbf{y}-\mathbf{H} \mathbf{x}\|^{2}+\mu\|\mathbf{D} \mathbf{x}\|^{2}+\nu\|\mathbf{E} \mathbf{x}\|^{2}
$$

where the first term measures the goodness of fit between the data $\mathbf{y}$ and the observation model for a candidate solution $\mathbf{x}$ and the second and third term regularize the solution, e.g. by penalizing large differences between spatially and/or spectrally adjacent pixels. Hyperparameters $\mu$ and $\nu$ are positive and balance the relative strength of the three terms of $\mathcal{J}$ (depending on the application and the level of blur, one of the parameters may be zero). $\mathbf{D}$ and $\mathbf{E}$ are respectively the spatial and spectral regularization matrices with $M^{2} N$ columns:

- For each channel $\ell$, spatial regularization is performed by selecting a convolution kernel $\mathcal{D}_{\ell}$ representing some kind of difference operator, e.g. the Laplacian filter:

$$
\left(\begin{array}{ccc}
0 & -1 & 0 \\
-1 & 4 & -1 \\
0 & -1 & 0
\end{array}\right)
$$

One can then construct the corresponding $M^{2} \times M^{2}$ CBC block $\mathbf{D}_{\ell}$ similar to the procedure given in section II-A. The hyperspectral spatial regularization matrix $\mathbf{D}$ has a block-diagonal structure:

$$
\mathbf{D}=\left[\begin{array}{cccc}
\mathbf{D}_{1} & \mathbf{0} & \ldots & \mathbf{0} \\
\mathbf{0} & \ddots & \ddots & \vdots \\
\vdots & \ddots & \ddots & \mathbf{0} \\
\mathbf{0} & \ldots & \mathbf{0} & \mathbf{D}_{N}
\end{array}\right] .
$$

- Spectral regularization occurs across channels, so matrix $\mathbf{E}$ is not block-diagonal. A simple choice to penalize large amplitude differences at adjacent wavelengths is to use a first order derivative filter $\mathcal{E}_{0}{ }^{1}$ in the wavelength domain, e.g. $\mathcal{E}_{0}=(1,-1)$, which yields the $1 \mathrm{D}$ convolution matrix:

$$
\mathbf{E}_{0}=\left(\begin{array}{ccccc}
-1 & 1 & 0 & \ldots & 0 \\
0 & -1 & \ddots & \ddots & \vdots \\
\vdots & \ddots & \ddots & \ddots & 0 \\
0 & \ddots & \ddots & -1 & 1
\end{array}\right)
$$

of size $(N-1) \times N$. The spectral regularization matrix reads

$$
\mathbf{E}=\mathbf{E}_{0} \otimes \mathbf{I}_{M}
$$

where $\mathbf{I}_{M}$ is the identity matrix of size $M \times M$, and $\otimes$ stands for the Kronecker product between matrices, consisting in replacing the 0's and 1's in (8) by $\mathbf{0}$ and $\mathbf{I}_{M}$, respectively.

The method proposed hereafter is not specific to the regularization operators defined in $(7-9)$; it can be applied to any convolution operators $\mathcal{D}_{\ell}$ 's with periodic boundary conditions and to any $\mathcal{E}_{0}$ (without imposing specific boundary conditions). Although matrices $\mathbf{D}$ and $\mathbf{E}$ are very large, they are mainly introduced for formal reasons. As we will see in Section IV, they are not explicitly stored in practice because the terms $\|\mathbf{D} \mathbf{x}\|^{2}$ and $\|\mathbf{E x}\|^{2}$ can be efficiently computed using two-dimensional Fourier transforms [12].

${ }^{1}$ Convolution kernel $\mathcal{E}_{0}$ is written with a subscript to remain coherent with other kernels $\mathcal{D}_{\ell}$

\section{B. Positive restoration problem}

The positive deconvolution problem is formulated in the framework of constrained optimization

$$
\min _{\mathbf{x}} \mathcal{J}(\mathbf{x}) \text { s.t. } \mathbf{x} \geq \mathbf{0}
$$

This is a classic constrained minimization problem which has no closed-form solution. Constrained optimization methods are usually classified as interior point, active set and exterior penalty methods [13]. The quadratic penalty method belongs to the latter class and is often used in computations for its simplicity [13, chapter 17]. The rationale for choosing this technique is that it allows us to use a slightly modified implementation of the unconstrained minimization of $\mathcal{J}$, based on fast computations in the Fourier domain, in order to yield the constrained estimate. This results in an algorithm with a relatively low complexity, which is especially useful when processing large hyperspectral images. The minimization procedure is detailed in section IV.

\section{NUMERICAL OPTIMIZATION}

The quadratic penalty method simply consists in replacing the constraints by quadratic penalty terms in the objective function. Handling inequality constraints can be done by introducing $M^{2} N$ slack variables $s_{i}$ and replacing the inequalities $\left\{x_{i} \geq 0, i=1 \ldots M^{2} N\right\}$ by $\left\{x_{i}-s_{i}=0, s_{i} \geq 0, i=1 \ldots M^{2} N\right\}$. The original problem (10) is replaced by the surrogate problem:

$$
\min _{\mathbf{x}, \mathbf{s}} \mathcal{K}(\mathbf{x}, \mathbf{s}) \text { s.t. } \mathbf{s} \geq \mathbf{0}
$$

where the new objective function reads

$$
\mathcal{K}(\mathbf{x}, \mathbf{s})=\|\mathbf{y}-\mathbf{H} \mathbf{x}\|^{2}+\mu\|\mathbf{D} \mathbf{x}\|^{2}+\nu\|\mathbf{E} \mathbf{x}\|^{2}+\xi\|\mathbf{x}-\mathbf{s}\|^{2} .
$$

When $\xi$ tends to infinity, the entries of $\mathbf{x}$ identify with the corresponding entries of $\mathbf{s}$ : the constraint $\mathbf{x} \geq \mathbf{0}$ is asymptotically fulfilled. The surrogate problem is unconstrained w.r.t. $\mathbf{x}$ and involves the constraint $\mathbf{s} \geq \mathbf{0}$. However, the positivy constraint is much easier to handle than in the original problem since the objective function is quadratic and separable w.r.t. s. Minimization of $\mathcal{K}$ w.r.t. $(\mathbf{x}, \mathbf{s})$ is performed by alternating unconstrained minimization w.r.t. $\mathbf{x}(\S \mathrm{IV}-\mathrm{A})$, constrained minimization w.r.t. s ( $(\mathrm{IV}-\mathrm{B})$ and increasing $\xi(\S \mathrm{IV}-\mathrm{C})$. The three steps are done once per iteration until some appropriate stopping criterion is met (§IV-D).

\section{A. Unconstrained minimization}

At a given iteration, the first step consists in solving the unconstrained quadratic minimization problem:

$$
\min _{\mathbf{x}} \mathcal{K}(\mathbf{x}, \mathbf{s})
$$

We first rewrite the objective function in terms of its channel components:

$$
\begin{aligned}
\mathcal{K}(\mathbf{X}, \mathbf{S})=\sum_{\ell=1}^{N} & \left(\left\|\mathbf{Y}_{\ell}-\mathcal{H}_{\ell} \underset{2 D}{*} \mathbf{X}_{\ell}\right\|^{2}+\mu\left\|\mathcal{D}_{\ell} \underset{2 D}{*} \mathbf{X}_{\ell}\right\|^{2}\right. \\
& \left.+\nu\left\|\left\{\mathcal{E}_{0} \underset{1 D}{*} \mathbf{X}\right\}_{\ell}\right\|^{2}+\xi\left\|\mathbf{X}_{\ell}-\mathbf{S}_{\ell}\right\|^{2}\right)
\end{aligned}
$$

where $*_{1 D}$ denotes the discrete $1 \mathrm{D}$ convolution product carried out in the spectral (wavelength) domain, i.e., there are as many 1D convolution products as the number of image pixels, and $\|\cdot\|$ is the matrix Frobenius norm. Using the 2D-DFT (denoted by the usual hat 
notation) and Parseval's Theorem, criterion (14) reads

$$
\begin{aligned}
\mathcal{K}^{\prime}(\hat{\mathbf{X}}, \hat{\mathbf{S}})=\sum_{\ell=1}^{N} & \left(\left\|\hat{\mathbf{Y}}_{\ell}-\hat{\mathcal{H}}_{\ell} \times \hat{\mathbf{X}}_{\ell}\right\|^{2}+\mu\left\|\hat{\mathcal{D}}_{\ell} \times \hat{\mathbf{X}}_{\ell}\right\|^{2}\right. \\
& \left.+\nu\left\|\left\{\mathcal{E}_{0} \underset{1 D}{*} \hat{\mathbf{X}}\right\}_{\ell}\right\|^{2}+\xi\left\|\hat{\mathbf{X}}_{\ell}-\hat{\mathbf{S}}_{\ell}\right\|^{2}\right)
\end{aligned}
$$

where $\times$ denotes entry-wise multiplication, $\|$.$\| now refers to the$ matrix Frobenius norm in the spatial frequency domain and $*_{1 D}$ still refers to the 1D convolution in the wavelength domain. To obtain (15), we have exploited that for a given $\ell,\left\{\mathcal{E}_{0} *_{1 D} \mathbf{X}\right\}_{\ell}$ is a linear combination of images $\left\{\mathbf{X}_{\ell^{\prime}}, \ell^{\prime}=1 \ldots N\right\}$ weighted by the nonzero values of $\mathcal{E}_{0}$. Thus, the 2D-DFT of $\left\{\mathcal{E}_{0} *_{1 D} \mathbf{X}\right\}_{\ell}$ is the linear combination of images $\hat{\mathbf{X}}_{\ell^{\prime}}$ 's with identical weights.

To ensure that all images $\mathbf{X}_{\ell}$ 's are real-valued, it is necessary to impose that $\hat{\mathbf{X}}_{\ell}$ 's are Hermitian matrices. Thus, it can readily be shown that the problem reduces to the minimization of $\mathcal{K}^{\prime}$ over half the 2D spatial frequency plane, e.g. the right-half plane and the whole minimizer is deduced using Hermitian symmetry.

We remark that in (15), each squared norm reads as a sum over the spatial frequencies $\mathbf{f}$ of the related matrix elements $(e . g$., the first term is the sum over $\mathbf{f}$ of $\left.\left|\hat{\mathbf{Y}}_{\ell}(\mathbf{f})-\hat{\mathcal{H}}_{\ell}(\mathbf{f}) \hat{\mathbf{X}}_{\ell}(\mathbf{f})\right|^{2}\right)$. Because the convolution $\left\{\mathcal{E}_{0} *_{1 D} \mathbf{X}\right\}_{\ell}$ is done in the wavelength domain, the objective function $\mathcal{K}^{\prime}(\hat{\mathbf{X}}, \hat{\mathbf{S}})$ is separable with respect to each spatial frequency $\mathbf{f}$. To be more specific, the problem may be recast as a collection of $\left\lfloor M^{2} N / 2\right\rfloor$ independent quadratic problems, each corresponding to a point $\mathbf{f}$ in the right space frequency half-plane, with $N$ unknowns:

$$
\min _{\mathbf{x}_{\mathbf{f}}}\left\{\left\|\mathbf{y}_{\mathbf{f}}-\boldsymbol{\Delta}_{\mathcal{H}}(\mathbf{f}) \mathbf{x}_{\mathbf{f}}\right\|^{2}+\mu\left\|\boldsymbol{\Delta}_{\mathcal{D}}(\mathbf{f}) \mathbf{x}_{\mathbf{f}}\right\|^{2}+\nu\left\|\mathbf{E}_{0} \mathbf{x}_{\mathbf{f}}\right\|^{2}+\xi\left\|\mathbf{x}_{\mathbf{f}}-\mathbf{s}_{\mathbf{f}}\right\|^{2}\right\}
$$

where complex vectors $\mathbf{x}_{\mathbf{f}}, \mathbf{y}_{\mathbf{f}}$ and $\mathbf{s}_{\mathbf{f}}$ and complex diagonal matrices $\boldsymbol{\Delta}_{\mathcal{H}}(\mathbf{f})$ and $\boldsymbol{\Delta}_{\mathcal{D}}(\mathbf{f})$ index the following quantities

$$
\begin{aligned}
\mathbf{x}_{\mathbf{f}} & \triangleq\left\{\hat{\mathbf{X}}_{\ell}(\mathbf{f}), \ell=1 \ldots N\right\} \\
\mathbf{y}_{\mathbf{f}} & \triangleq\left\{\hat{\mathbf{Y}}_{\ell}(\mathbf{f}), \ell=1 \ldots N\right\} \\
\mathbf{s}_{\mathbf{f}} & \triangleq\left\{\hat{\mathbf{S}}_{\ell}(\mathbf{f}), \ell=1 \ldots N\right\} \\
\boldsymbol{\Delta}_{\mathcal{H}}(\mathbf{f}) & \triangleq \operatorname{diag}\left\{\hat{\mathcal{H}}_{\ell}(\mathbf{f}), \ell=1 \ldots N\right\} \\
\boldsymbol{\Delta}_{\mathcal{D}}(\mathbf{f}) & \triangleq \operatorname{diag}\left\{\hat{\mathcal{D}}_{\ell}(\mathbf{f}), \ell=1 \ldots N\right\}
\end{aligned}
$$

and $\mathbf{E}_{0}$ is the $1 \mathrm{D}$ convolution matrix induced by kernel $\mathcal{E}_{0}$. For each $\mathbf{f}$ in the right half-plane, the solution of (16) is then simply given by

$$
\mathbf{x}_{\mathbf{f}}=\mathbf{T}_{\mathbf{f}}{ }^{-1}\left(\boldsymbol{\Delta}_{\mathcal{H}}(\mathbf{f})^{\prime} \mathbf{y}_{\mathbf{f}}+\xi \mathbf{s}_{\mathbf{f}}\right)
$$

where ' stands for the complex conjugate and $\mathbf{T}_{\mathbf{f}}$ is the real $N \times N$ matrix given by

$$
\mathbf{T}_{\mathbf{f}}=\boldsymbol{\Delta}_{\mathcal{H}}(\mathbf{f})^{\prime} \boldsymbol{\Delta}_{\mathcal{H}}(\mathbf{f})+\mu \boldsymbol{\Delta}_{\mathcal{D}}(\mathbf{f})^{\prime} \boldsymbol{\Delta}_{\mathcal{D}}(\mathbf{f})+\nu \mathbf{E}_{0}^{t} \mathbf{E}_{0}+\xi \mathbf{I}_{N} .
$$

Choosing $\mathcal{E}_{0}=(1,-1)$ leads to (8) which implies that $\mathbf{T}_{\mathbf{f}}$ is a tridiagonal matrix.

\section{B. Constrained minimization}

The second step consists in solving the following separable quadratic minimization problem:

$$
\min _{\mathbf{s} \geq 0} \mathcal{K}(\mathbf{x}, \mathbf{s})
$$

where the positivity constraint on $\mathbf{s}$ is easy to handle since the only term of $\mathcal{K}$ in which $\mathbf{s}$ is involved is the last term $\|\mathbf{x}-\mathbf{s}\|^{2}$. This term is separable with respect to $s_{i}$ 's and the solution reads

$$
s_{i}=\max \left(0, x_{i}\right) .
$$

\section{Update of $\xi$}

Increasing $\xi$ at each iteration ensures that the algorithm converges to the constrained minimizer of $\mathcal{J}$. A broad range of schemes is available and a common choice is to use a linear rule $\xi^{(k+1)}=\alpha \xi^{(k)}$ where $\alpha>1$ [13, p. 493].

\section{Proposed algorithm}

The algorithm procedure can be summed up as follows ( $\tau$ is a stopping parameter):

1) for $\ell=1 \ldots N$, compute the 2D-DFTs of data $\mathbf{Y}_{\ell}$ and PSFs $\mathcal{H}_{\ell}\left(\mathcal{H}_{\ell}\right.$ 's are usually theoretically known or experimentally measured); set $\xi^{(1)}=1$ and $\alpha>1$ and $\tau<0$; initialize $\mathbf{s}=\mathbf{0}$;

2) repeat

a) for each $\ell$, compute $\mathbf{S}_{\ell}$ from $\mathbf{s}_{\ell}$ using $2 \mathrm{D}-\mathrm{FFT}$;

b) for each $\mathbf{f}$ in the right-half plane, compute $\left\{\hat{\mathbf{X}}_{\ell}(\mathbf{f}), \ell=\right.$ $1 \ldots N\}$ using (17) and (18);

c) for each $\mathbf{f}$ in the left-half plane, deduce $\hat{\mathbf{X}}_{\ell}(\mathbf{f})$ 's using Hermitian symmetry;

d) for each $\ell$, compute $\mathbf{x}_{\ell}$ from $\hat{\mathbf{X}}_{\ell}$ using inverse 2D-FFTs;

e) compute $\mathbf{s}$ from (20);

f) update coefficient $\xi^{(k+1)}=\alpha \xi^{(k)}$.

until the stopping criterion is met, e.g. the lowest-valued pixel of $\mathbf{X}$ is superior to some tolerance threshold $\tau$.

\section{E. Computational complexity}

Using the FFT, the complexity of a 2D-DFT is $\mathcal{O}\left(M^{2} \log _{2}(M)\right)$. The complexity of each iteration is dominated by FFTs since each $N \times N$ tridiagonal linear inversion reduces to $\mathcal{O}(N)$ using the Crout's LU decomposition algorithm [14]. Hence, each iteration is done in $\mathcal{O}\left(N M^{2} \log _{2}(M)\right)$ operations.

Additionally, no expensive storage is required since the largest matrix involved in computation $\mathbf{T}_{\mathbf{f}}$ is sparse and of size $N \times N$.

\section{EXPERIMENTAL RESULTS}

We illustrate the performance of the algorithm with experimental results on synthetic data. We use a linear mixing model to generate a multichannel cube of data representing simulated objects against a uniform background, captured at several wavelengths. In this model, the spectrum of one pixel $(i, j)$ is given by

$$
\mathbf{X}_{\ell}(i, j)=a_{1}(i, j) \mathbf{u}_{1}(\ell)+a_{2}(i, j) \mathbf{u}_{2}(\ell)
$$

where endmember $\mathbf{u}_{1}$ is the segment $[0,1]$ sampled on $N$ points and $\mathbf{u}_{2}$ is the vector obtained by flipping $\mathbf{u}_{1}$ in the left - right direction. By setting the corresponding abundances $a_{1}$ and $a_{2}$ to an arbitrary $M \times M$ spatial pattern, one may synthesize objects that respectively fade in and out when the channel index $\ell$ increases from 1 to $N$. Blurring is simulated by convolving each channel of the image by a $M \times M$ Airy disc computed by the 2 D DFT of a disk whose radius linearly depends on the channel $\ell$. The stack is further corrupted by an additive white Gaussian noise term so as to obtain a SNR of 15 $\mathrm{dB}$. The resulting $M \times M \times N$ images are not meant to be realistic but provide a simple way to evaluate the performance of the algorithm from a computational standpoint.

\section{A. Comparison with other constrained optimization methods}

In this section we investigate constrained optimization methods to solve problem (10). Nonnegativity constraints are most commonly handled by the log-barrier interior point method [15], [13]. We use 


\begin{tabular}{|r|r|r||c|c|c|c|}
\hline $\mathrm{M}$ & $\mathrm{N}$ & Variables & Proposed method & fmincon & quadprog & Unc. estimate \\
\hline \hline 25 & 3 & 1875 & $.6 \mathrm{~s}$ & $510.9 \mathrm{~s}$ & $1.6 \mathrm{~s}$ & $.1 \mathrm{~s}$ \\
\hline 50 & 5 & 12500 & $1.9 \mathrm{~s}$ & $3863.6 \mathrm{~s}$ & $11.6 \mathrm{~s}$ & $.2 \mathrm{~s}$ \\
\hline 100 & 10 & 100000 & $9.2 \mathrm{~s}$ & manually stopped after 2 hours & memory limitation & $1.0 \mathrm{~s}$ \\
\hline
\end{tabular}

TABLE I

COMPARISON OF THE COMPUTATION TIMES OF THE DIFFERENT OPTIMIZATION METHODS

the MATLAB optimization toolbox to write two different implementations of this technique, using either matrices with the quadprog function and a Fourier domain formulation with fmincon. We also compare these results with the unconstrained estimate [12]. A pair of hyperparameters is arbitrarily selected and results are given for a 2.4 Ghz Intel Core 2 Duo processor with a RAM of 4 gigabytes.

The unconstrained estimate performs worse in terms of MSE (defined as $\left\|\mathbf{x}_{\text {true }}-\mathbf{x}_{\text {est }}\right\|_{2}^{2}$ ) : see figure 1. We observe that all three constrained optimization methods do converge to the same estimate, yielding very similar objective values and identical reconstruction errors. However, our method strongly outperforms the others in terms of computation time and memory requirements, as shown in table I.

The main reason for the increased computation time is that the log-barrier objective function is not quadratic, hence its gradient is nonlinear and thus minimizing the objective w.r.t. to $\mathbf{x}$ must be done in an iterative fashion, e.g. using a quasi-Newton step. When the data size is large, closed-form minimizations at each iteration make the restoration problem tractable. Furthermore, we observe that computing convolution matrices quickly reaches memory limitations.

\section{B. Sensitivity to the choice of hyperparameters}

Running the algorithm involves choosing the hyperparameter pair $(\mu, \nu)$. Because of the tradeoff between spatial and spectral regularization and their interplay with the positivity constraint, this is not a trivial problem and its analytical resolution is still an open problem. Furthermore, the choice strongly depends on the application, e.g. the restored image should minimize the reconstruction error as a preprocessing step to spectral unmixing, but applying more smoothing (hence selecting higher parameters) is often desirable for visual inspection. In this section we select the MSE as the relevant criterion. We attribute a $\left[10^{-4}, 10^{4}\right]$ range to each hyperparameter and discretize it on a 2D grid with logarithmic scales. For each grid point, we restore the data using the proposed method and we compute the MSE. The results corresponding to the least MSEs are presented in figure 1 for $M=25$ and $N=3$.

We observe that the best constrained estimate in the MSE sense is obtained for lower hyperparameters, i.e. requires less smoothing than the best unconstrained estimate: see for instance [16]. In this simulation, the estimation is not very sensitive to the variation of hyperparameters, the ratio between maximum and minimum MSEs on the grid being 1.3 for the constrained and 1.6 for the unconstrained estimate. What is more, the former has a lower reconstruction error than the latter and presents slightly less restoration artifacts: see figure 1. Notice how more ringing artifacts are located near the edges of the unconstrained estimate as opposed to the more "uniform" distribution of errors for the constrained estimate.

In practical applications we obviously do not have access to the true image and hyperparameters may best be selected by trial and error. However, this process remains tractable in practice since the proposed method remains fast even for large data and numerous hyperparameter candidate values.

\section{C. $3 D+\lambda$ Fluorescence microscopy simulation}

In this section we demonstrate the performance of the algorithm on synthetic data resembling actual fluorescence microscopy images for visual inspection and to get an idea of the processing time of 4D data for a MATLAB implementation. We create 3D abundance maps of physical size $25 \mu \mathrm{m} \times 25 \mu \mathrm{m} \times 5 \mu \mathrm{m}$ to mimic the random spatial distribution of bacteria using spheres of $1 \mu \mathrm{m}$ radius, yielding $255 \times 255 \times 5$ data cubes. Pseudo fluorescent spectra modeled as sums of Gaussian signals in the $300-600 \mathrm{~nm}$ range are fed into the linear mixing model to create the $255 \times 255 \times 5 \times 30$ data stack. We use the scalar diffraction-limited Stokseth model [17] to generate a widefield microscopy point-spread function with a $63 \mathrm{X} / 1.4$ oil immersion objective and a CCD camera pixel size of $0.1 \mu \mathrm{m}$. The image is blurred and an additive white Gaussian noise term is added to obtain a SNR of $15 \mathrm{~dB}$ : in widefield microscopy, the SNR is high enough so that the Poisson distributed photo-counting noise term can be accurately approximated by a Gaussian process [17]. Results are presented in figure 2. Due to the use of $4 \mathrm{D}$ tensors, the running time has increased to $15 \mathrm{~min}$ for ten iterations of the restoration algorithm. Deconvolution achieves a $77 \%$ decrease of the MSE and the proposed estimate thus has a significantly greater resolution. By using information from neighboring wavelengths, our method can restore severely degraded image planes which cannot be tackled by simpler schemes such as PSF fitting.

\section{CONCLUSION}

This paper presents a method to solve a hyperspectral image deconvolution problem where one needs to jointly account for spatial, spectral and non-negativity information. The problem is formulated as the minimization of a regularized least-squares criterion under a positivity constraint. While a closed-form solution to the unconstrained problem is known, we provide an intuitive implementation based on FFTs. We show how to easily incorporate a positivity constraint into the estimate. The main advantages of the method are its ease of implementation since it does not require computation of large matrices and its speed, mainly due to the use of 2DFFTs and low-complexity tridiagonal inversions. Furthermore, the algorithm can make use of parallel processing on each pixel of the stack of spectra using Graphics Processing Units (GPUs) [18] with no additional implementation issues. The method can also be extended to hyperspectral images with three spatial dimensions in a straightforward manner. Future investigations might include accounting for Poisson noise in accordance with confocal microscopy observation models.

\section{ACKNOWLEDGMENT}

The authors thank Dr. S. Moussaoui and Prof. J. Idier from IRCCyN (UMR CNRS 7597, Nantes, France) for fruitful discussions regarding interior point algorithms.

\section{REFERENCES}

[1] P. Sarder and A. Nehorai, "Deconvolution methods for 3D fluorescence microscopy images," IEEE Sig. Proc. Mag, vol. 23, pp. 32-45, 2006. 
True image
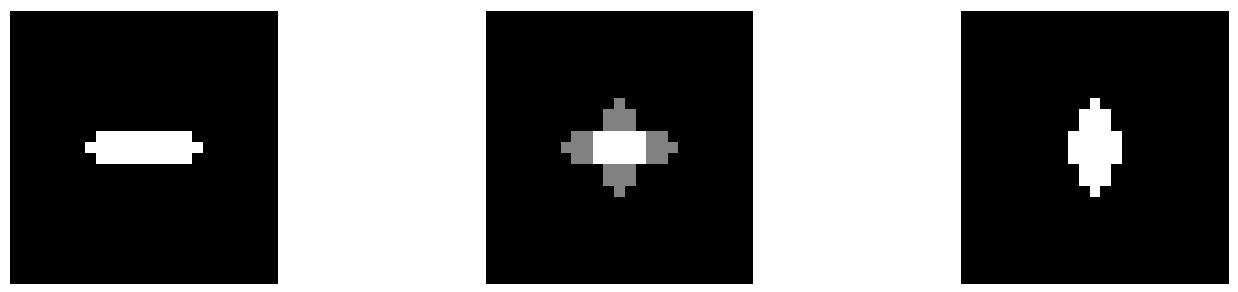

Degraded image

$\mathrm{RMSE}=4.2 e^{-3}$
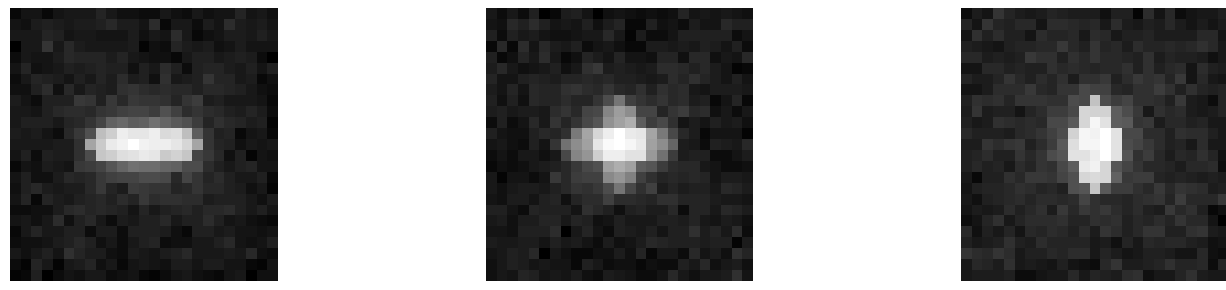

Constrained estimate

RMSE $=1.8 e^{-3}$
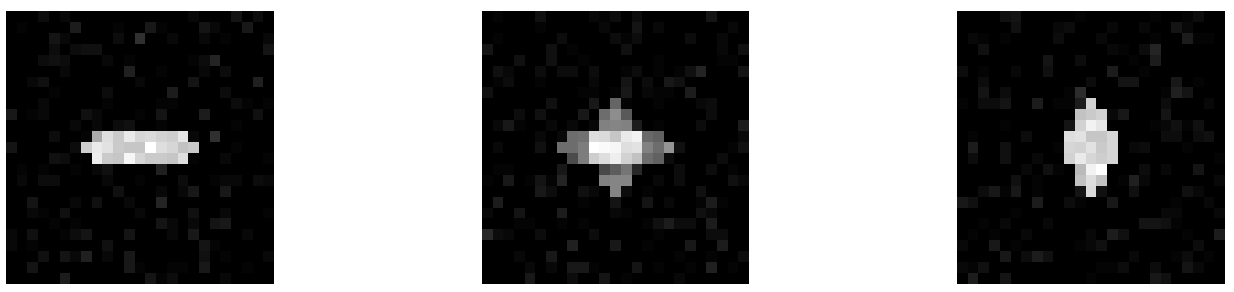

Reconstruction error
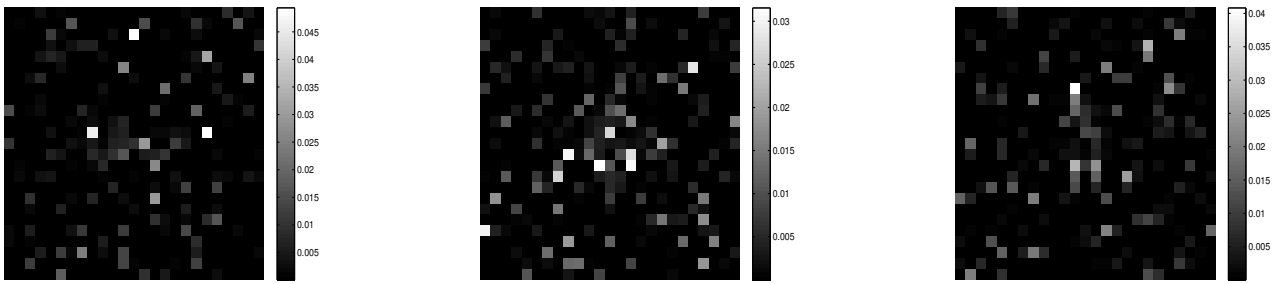

\section{Unconstrained estimate}

$\mathrm{RMSE}=2.2 e^{-3}$
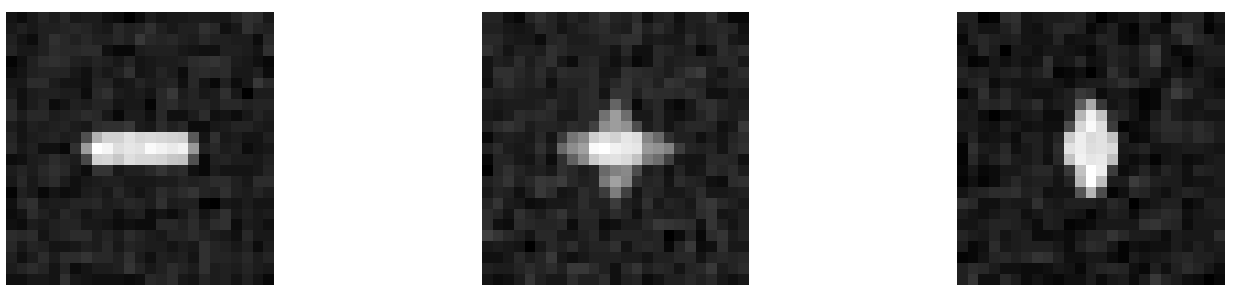

Reconstruction error
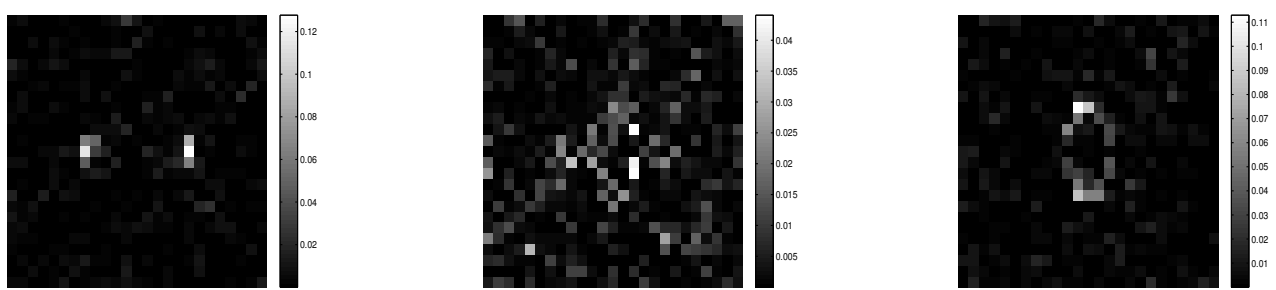

Fig. 1. True, degraded and restored $25 \times 25 \times 3$ toy images for a SNR of 15. Each column corresponds to a channel. 
Degraded image

$\mathrm{RMSE}=1.2 e^{-2}$
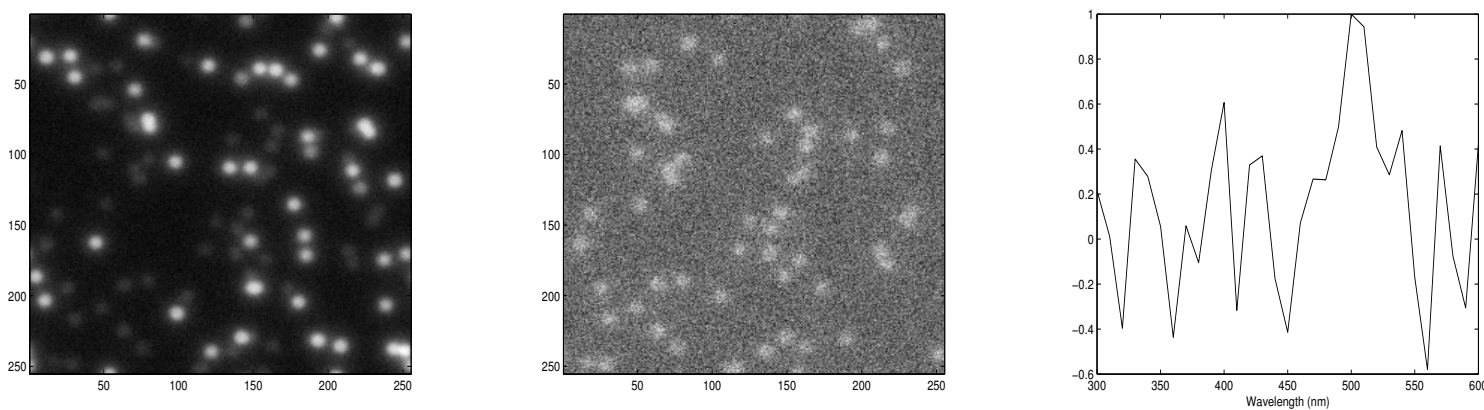

\section{Constrained estimate}

$\mathrm{RMSE}=2.3 e^{-3}$
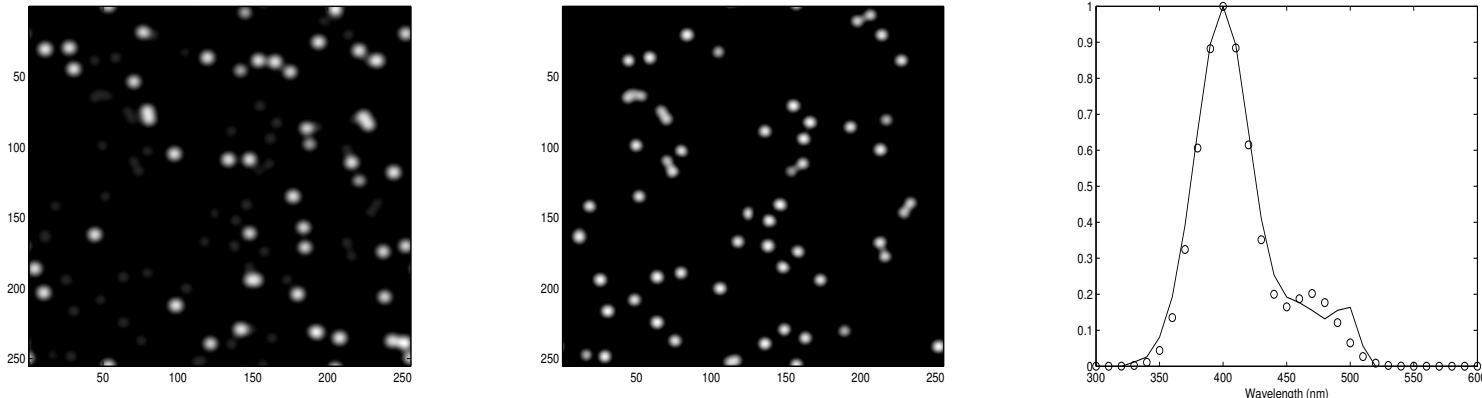

Fig. 2. Degraded and restored $255 \times 255 \times 5 \times 30$ synthetic fluorescent microscopy images for a SNR of 15 . The first two columns correspond to a channel. The last column displays the observed spectrum of a pure pixel belonging to source $\mathbf{u}_{\mathbf{1}}$ (first row) and the spectrum retrieved by the algorithm (second row, dots indicate the true source spectrum $\mathbf{u}_{1}$ ).

[2] Y. Zhang, A. Duijster, and P. Scheunders, "A hyperspectral image restoration technique," in Proceedings of the 16th IEEE International Conference on Image Processing (ICIP), 2009.

[3] S. Bongard, F. Soulez, E. Thibaut, and E. Pecontal, "3D deconvolution of hyper-spectral astronomical data," Monthly Notices of the Royal Astronomical Society, vol. 418, pp. 258270, 2011.

[4] N. P. Galatsanos and R. T. Chin, "Digital restoration of multichannel images," IEEE Trans. Acous., Speech, Signal Processing, vol. 37, pp. 415-421, 1989.

[5] N. P. Galatsanos, A. K. Katsaggelos, R. T. Chin, and A. D. Hillery, "Least squares restoration of multichannel images," IEEE Trans. Signal Processing, vol. 39, pp. 2222-2236, 1991.

[6] E. Thiébaut, "Introduction to image reconstruction and inverse problems," in Proceedings of the NATO Advanced Study Institute on Optics in Astrophysics, 2005.

[7] S. Shah, Deconvolution algorithms for fluorescence and electron microscopy, Ph.D. dissertation, University of Michigan, 2006.

[8] J.-F. Giovannelli and A. Coulais, "Positive deconvolution for superimposed extended source and point sources," Astronomy and Astrophysics, vol. 439, pp. 401-412, 2005.

[9] M. Afonso, J. Bioucas-Dias, and M. Figueiredo, "Fast image recovery using variable splitting and constrained optimization," IEEE Transactions on Image Processing, vol. 19, no. 9, pp. 2345-2356, 2010.

[10] F. Benvenuto, R. Zanella, L. Zanni, and M. Bertero, "Nonnegative leastsquares image deblurring: improved gradient projection approaches," Inverse Problems, vol. 26, pp. 1-18, 2010.

[11] M. Bertero, P. Boccacci, G. Desiderà, and G. Vicidomini, "Image deblurring with Poisson data: from cells to galaxies," Inverse Problems, vol. 12, pp. 1-26, Dec. 2009.

[12] S. Henrot, C. Soussen, and D. Brie, "Fast deconvolution of large hyperspectral images," in Proceedings of WHISPERS - 3rd Workshop on Hyperspectral Image and Signal Processing: Evolution in Remote Sensing, 2011.

[13] J. Nocedal and S. Wright, Numerical Optimization, Springer, 2006.

[14] W. H. Press, S. A. Teukolsky, W. T. Vetterling, and B. P. Flannery, Numerical recipes: The art of scientific computing, Cambridge Univ. Press, New York, 3rd edition, 2007.
[15] E. Chouzenoux, S. Moussaoui, and J. Idier, "Efficiency of liensearch strategies in interior point methods for linearly constrained signal restoration," in Proc. IEEE workshop on Statistical Signal Processing Workshop (SSP'11), Nice, France, 2011, pp. $101-104$.

[16] J. Bardsley, J. Merikoski, and R. Vio, "The stabilizing properties of nonnegativity constraints in least-squares image reconstruction," Int. J. of Pure and Applied Mathematics, vol. 43, no. 1, pp. 95-109, 2008.

[17] P. Pankajakshan, Blind Deconvolution for Confocal Laser Scanning Microscopy, Ph.D. dissertation, Université de Nice Sophia-Antipolis, 2009.

[18] A. Plaza, J. Plaza, A. Paz, and S. Sanchez, "Parallel hyperspectral image and signal processing," IEEE Signal Processing Magazine, vol. 28, pp. $119-126,2011$.

Simon Henrot was born in France in 1986. He received the diploma degree in electrical engineering from the Ecole Nationale de l'Aviation Civile, Toulouse, France in 2009 and is currently a Ph.D. student in signal processing with the Centre de Recherche en Automatique de Nancy and the Université de Lorraine, Nancy, France.

Charles Soussen was born in France in 1972. He received the degree from the École Nationale Supérieure en Informatique et Mathématiques Appliquées, Grenoble, France, and the Ph.D. degree in Physics from the Laboratoire des Signaux et Systèmes, Université de Paris-Sud, Orsay, France, in 1996 and 2000, respectively. He is currently an Assistant Professor at Université de Lorraine, France. He has been with the Centre de Recherche en Automatique de Nancy since 2005. His research interests are in inverse problems and sparse approximation.

David Brie received the Ph.D. degree in 1992 and the Habilitation à diriger des Recherches degree in 2000, both from the Henri Poincaré University, Nancy, France. He is currently Professor at the telecommunication and network department from the Institut Universitaire de Technologie, Université de Lorraine. Since 1990, he has been with the Centre de Recherche en Automatique de Nancy. His research interests mainly concern inverse problems and multidimensional signal processing. 